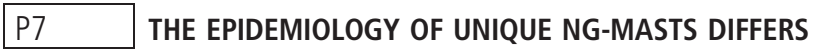
FROM THAT OF COMMONLY CIRCULATING NG-MASTS IN LOTHIAN, SCOTLAND

Kate Mitchell*, Rachel Manners, Kirstine Eastick, Imali Fernando*. NHS Lothian, Edinburgh, UK

10.1136/sextrans-2015-052126.51

Background/introduction Surveillance of gonococcal infection in Scotland has demonstrated variation in the prevalence of Neisseria gonorrhoeae multi-antigen sequence types. Some appear to circulate extensively, other sequence types (STs) may be recorded only once.

Aim(s)/objectives We aimed to review the epidemiology of gonococcal infection resulting from unique STs and examine for any association between unique STs and antibiotic resistance patterns.

Methods All gonococcal isolates from Lothian with a ST unique to Scotland, identified July 2006-October 2013, were included in the study. A control group of 76 patients infected with commonly circulating STs was also identified from the same study period.

Results 92 cases of N. gonorrhoae with unique STs were identified. Of these, 55 were truly distinct and categorised as 'unique and different'. The remaining 33 cases had STs which differed only slightly from locally circulating types and were likely to have evolved due to mutation of common strains. These were categorised as 'unique and similar'. Patients infected with 'unique and different' STs were significantly more likely than controls $(\mathrm{p}<0.5)$ to have had recent sexual contact outside of Europe and/or had a recent sexual partner of non-British nationality. However, they were no more likely to have a significantly altered antibiotic resistance profile, though there was a trend towards increased antibiotic resistance.

Discussion/conclusion Gonococcal strains from non-European countries may be associated with antibiotic resistance. Identification of a 'unique and different' sequence type raises the possibility of an imported strain of gonococcal infection and demands particular vigilance in looking for antibiotic resistance.

\section{P8 WHERE DO PATIENTS GO FOR CHLAMYDIA TESTING WITHIN NON-GUM COMMUNITY SETTINGS AND WHAT PROPORTION OF RE-TESTERS SHOWS VENUE LOYALTY?}

Ana Harb*, Katy Town, Emma Hollis, Catherine Lowndes, Kevin Dunbar. Public Health England, London, UK

\subsection{6/sextrans-2015-052126.52}

Background/introduction The English National Chlamydia Screening Programme focuses on prevention, control and treatment of chlamydia in sexually active under- 25 year olds. A greater understanding of where young adults attend services helps to inform commissioners of where to focus resources within community settings.

Aim(s)/objectives To investigate whether young people return to the same type of primary care / community (i.e. non-Genitourinary Medicine) service for re-testing.

Methods Surveillance data from the Chlamydia Testing Activity Dataset (CTAD) was used to identify patient attendances at primary care / non GUM community services among 15 to 24 year olds and monitor re-testing within and between community services.

Results From January 2012 to December 2013, 1,333,718 young people underwent 1,626,106 chlamydia tests. The majority of people $(84 \%)$ were tested only once. Of those who tested more than once, $57 \%$ used the same venue type. General Practice (GP) was the most commonly re-attended service for patients who tested twice (55.3\%). Among those who tested three or four times, there was an increasing preference for community sexual health services $(50 \%$ and $57 \%$ respectively).

Discussion Patients re-attended GP services more often than other venue types but for subsequent attendances more specialised community sexual health services were used. Very few repeat visits were made to pharmacies or pregnancy termination services. These data show that patients are likely to return to services they know when they require a further test. This should be taken into consideration by commissioners implementing new retesting guidance from the NCSP.

\section{P9 LOW FALSE POSITIVE RATE OF GONORRHOEA CASES REFERRED TO GUM FROM A LOW PREVALENCE CHLAMYDIA SCREENING POPULATION}

${ }^{1}$ Agnieszka Tan*, ${ }^{1}$ Stephen Brolly, ${ }^{2}$ Neil Howard, ${ }^{1}$ Samir Dervisevic, ${ }^{1}$ Jo Evans. ${ }^{1}$ Norfolk and Norwich University Hospitals NHS Foundation Trust, Norwich/Norfolk, UK; ${ }^{2}$ Norfolk and Waveney Chlamydia Screening Programme, Norwich/Norfolk, UK

\subsection{6/sextrans-2015-052126.53}

Background/introduction Recent national guidance for the detection of gonorrhoea (GC) has raised concerns that the majority of initial positive GC test results are likely to be false positives when a low prevalence population is screened using a nucleic acid amplification test (NAAT). Our local chlamydia screening programme uses a dual NAAT for chlamydia and GC and has a low reactive GC rate $<1 \%$. All GC reactive cases are referred to GUM for further investigation.

Aim(s)/objectives To determine whether GC reactive cases referred from our local chlamydia screening programme were true GC infections or likely to be false positive cases.

Methods A retrospective case notes review of 13 consecutive GC reactive cases seen at our clinic referred from the local chlamydia screening programme.

Results 10/13 were women. 10/13 cases were confirmed as true GC infections by positive genital GC cultures. In 1 case genital culture was negative but screening and supplementary NAAT with a different target confirmed a true GC infection. In 1 case genital cultures were negative however pharyngeal culture was positive indicating a true GC infection. In 1 case GC cultures were all negative but NAAT tests were reactive from genital and pharyngeal sites and equivocal from the rectum.

Discussion/conclusion Only 1/13 GC reactive cases seen in our GU referred from a low prevalence screening population might have been a false positive. Contrary to recent publications, in our area, using a dual NAAT is unlikely to lead to high numbers of false positive GC results. 